“( 2014 IEEE. Personal use of this material is permitted. Permission from IEEE must be obtained for all other uses, in any current or future media, including

reprinting/republishing this material for advertising or promotional purposes, creating new collective works, for resale or redistribution to servers or lists, or reuse of any copyrighted component of this work in other works." 


\title{
Neural Network Based Diagonal Decoupling Control of Powered Wheelchair Systems
}

\author{
Tuan Nghia Nguyen, Member, IEEE, Steven Su, Member, IEEE and Hung T Nguyen, Senior Member, IEEE
}

\begin{abstract}
This paper proposes an advanced diagonal decoupling control method for powered wheelchair systems. This control method is based on a combination of the systematic diagonalization technique and the neural network control design. As such, this control method reduces coupling effects on a multivariable system, leading to independent control design procedures. Using an obtained dynamic model, the problem of the plant's Jacobian calculation is eliminated in a neural network control design. The effectiveness of the proposed control method is verified in a real-time implementation on a powered wheelchair system. The obtained results confirm that robustness and desired performance of the overall system are guaranteed, even under parameter uncertainty effects.
\end{abstract}

Index Terms-Multivariable Control System, Diagonalization Technique, Neural Network Control, Powered Wheelchair.

\section{INTRODUCTION}

Powered wheelchairs are increasingly important, as the number of people with severe motor impairment increases. However, safety control of a conventional power wheelchair requires a significant level of skill, attention, judgment and appropriate behavior. Furthermore, wheelchair users may also encounter accidents and injuries, similar to automobile users. Every year in the United States, around 85,000 serious wheelchair accidents occur, and the trend is expected to increase [1].

Much research has been conducted in the development of autonomous and semi-autonomous capabilities for allowing a wheelchair to perform independently, or in combination with the user, to sustain automatic functionalities such as obstacle avoidance, following corridors, or passing doors. These functionalities aid in the provision of enhanced safety and comfort for severely handicapped wheelchair users. Worldwide a lot of progress has been made in the development of wheelchair automatism. Nevertheless, the majority of research activity focuses on the level of supervisory control. This supervisory level is involved in developing navigation algorithms, shared-control approaches, and hands-free control strategies. Although low control level (or drive control level), which is involved in precision motion control, guarantees the desired performance and stability of the overall system, research on control techniques for this level is still largely lacking.

A dynamic wheelchair model is required for developing control algorithms in the low control level. However, due to parametric variations caused by wheelchair mechanisms and the operating environment, it is difficult if not impossible to

The authors are with Faculty of Engineering and Information Technology, Sydney, NSW 2007, Australia (email: tuannghia.nguyen@uts.edu.au; steven.su@uts.edu.au; hung.nguyen@uts.edu.au). obtain an exact dynamic model for the powered wheelchair. Only a steering model (kinematic model), derived from geometry constraints, is used to develop motion control algorithms. These constraints state that the wheelchair can only move in the direction which is normal to the axis of the driving wheels. As a result, methods based on a kinematic model may not be practical. This is because physical parameters, surface friction coefficients, and mass, all significantly affect performance and stability of the overall system. Furthermore, the literature on robustness and control in the presence of uncertainties in the dynamical model of such systems is meager [2].

In terms of low level control design, many advanced control techniques have already been developed [3]-[8]. However, most of these have not treated the powered wheelchair as a multivariable system, and are therefore unlikely to be optimal. Due to the interactions between different inputs and outputs, the implementation of a control design task can be complicated for a multivariable system. Decoupling techniques are very effective solutions to the issues inherent in a multivariable system. These techniques endeavour to simplify a multivariable control problem by reducing it to a series of scalar control problems [9]. However, research investigating the system robustness under the effects of uncertainty and external disturbance, is relatively scarce.

Although neural networks have provided effective solutions to complex and nonlinear control problems [10]-[12], they face the problem of an unknown plant Jacobian, this being defined as the partial derivative of a plant's outputs with respect to its inputs. This is applied when propagating the control error using the back propagation method to the adjustable coefficients of the neural controller. This coefficients' adjustment leads to error reduction. Due to the complexity of an unknown plant, it is difficult or even impossible to calculate a plant Jacobian. Several methods have been proposed for finding a plant Jacobian, though none have proven effectiveness. These methods include the online approximation method [13], the plant Jacobian sign calculation [14], direct calculation from the plant model [15], the Inverse Transfer Matrix scheme [10], gain layer schemes [16], and the use of trained neural networks as an identifier [15].

Recently, we have proposed a new advanced robust multivariable control strategy for a powered wheelchair system [17]. This control strategy is based on a combination of the systematic triangularization technique and the robust neurosliding mode control approach. This strategy effectively copes with parameter uncertainties and external disturbances in realtime in order to achieve robustness and optimal performance of a multivariable system. In this paper, we propose a new 
diagonalization technique, which completely eliminates the decoupling effects in a multivariable system. Moreover, a new neural network control design is also presented in this paper to prove the effectiveness of the proposed control strategy. This design utilizes the wheelchair dynamics model to calculate a plant Jacobian directly from the transfer function matrix. Thus, the problem of the plant Jacobian calculation is eliminated. More detail of wheelchair dynamics modeling is also presented in this paper.

In section II of this paper, a dynamic wheelchair system model is obtained. The diagonal decoupling technique for a multivariable system is described in Section III of this paper. In section IV, the neural network control design is presented in detail. Real-time experimental results and discussions are presented in section V. The conclusion is found in section VI.

\section{DyNAMics MODELling OF A POWERED WHEELCHAIR}

\section{A. Dynamic model of a powered wheelchair}

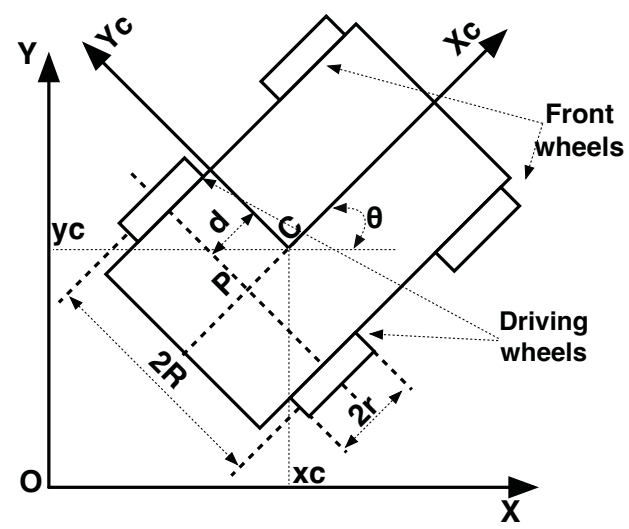

Fig. 1. The wheelchair system.

The wheelchair system is shown in the Fig. 1. It consists of a wheelchair with two driving wheels mounted in the same axis and two front wheels. The driving wheels are independently driven by two actuators to achieve motion and orientation. Both wheels have the same radius denoted by $r$. The distance between the two driving wheels is $2 R$. The center of mass of the wheelchair is located at $C$; point $P$ is located in the intersection of a straight line passing through the middle of the vehicle and a section which is an axis of the two wheels. The distance between points $P$ and $C$ is denoted by $d$. The pose of the wheelchair in the global coordinator frame $\{O, X, Y\}$ is completely specified by vector $q=\left[\begin{array}{lll}x_{c} & y_{c} & \theta\end{array}\right]^{T}$, where $x_{c}$ and $y_{c}$ are the coordinates of the point $C$ in the global coordinate frame and $\theta$ is the orientation of the local frame $\left\{C, X_{x}, Y_{x}\right\}$ attached on the wheelchair platform measured from the $X$ axis. Denoting the wheelchair mass by $m$ and wheelchair inertia by $I$, the complete dynamic equation of the wheelchair can be derived from [18] as follows

$$
\left[\begin{array}{cc}
m & 0 \\
0 & I-m d^{2}
\end{array}\right]\left[\begin{array}{c}
\dot{v} \\
\dot{\omega}
\end{array}\right]+\left[\begin{array}{c}
\bar{\tau}_{d r} \\
\bar{\tau}_{d l}
\end{array}\right]=\frac{1}{r}\left[\begin{array}{cc}
1 & 1 \\
R & -R
\end{array}\right]\left[\begin{array}{c}
\tau_{r} \\
\tau_{l}
\end{array}\right]
$$

where $\tau_{r}$ and $\tau_{l}$ respectively represent right and left wheel torques, disturbance torque vector $\left[\begin{array}{cc}\bar{\tau}_{d r} & \bar{\tau}_{d l}\end{array}\right]^{T}$ is assumed to be bounded, and velocity vector $\mathbf{v}=\left[\begin{array}{ll}v & \omega\end{array}\right]^{T}$ in which $v$ is linear velocity and $\omega$ is angular velocity. Assuming that both driven actuator motors are dc motors and neglecting motor inductance, the equation governing the actuator motors can be written as

$$
\left[\begin{array}{c}
\tau_{s r} \\
\tau_{s l}
\end{array}\right]=\left[\begin{array}{c}
K_{T r} i_{r} \\
K_{T l} i_{l}
\end{array}\right]
$$

where $\tau_{s r}$ and $\tau_{s l}$ are the torque generated by the respective right and left motors, $K_{T r}$ and $K_{T l}$ are the right and left motors' torque constants, and $i_{r}$ and $i_{l}$ are the current flowing in the right and left motors' coils,

$$
\left[\begin{array}{c}
u_{r} \\
u_{l}
\end{array}\right]=\left[\begin{array}{c}
R_{a r} i_{r}+K_{b r} \dot{\theta}_{r m} \\
R_{a l} i_{l}+K_{b l} \dot{\theta}_{l m}
\end{array}\right]
$$

where $R_{a r}$ and $R_{a l}$ are the respective right and left motors' resistances, $K_{b r}$ and $K_{b l}$ are the right and left counter electromotive force coefficients, and $\dot{\theta}_{r m}$ and $\dot{\theta}_{l m}$ represent the velocity of the right and left actuator motors, respectively.

The angular velocities of the actuator motors, $\dot{\theta}_{r m}$ and $\dot{\theta}_{l m}$, and the corresponding wheels' angular velocities, $\dot{\phi}_{r}$ and $\dot{\phi}_{l}$, are related by gear ratio $N$ as

$$
\left[\begin{array}{c}
\dot{\phi}_{r} \\
\dot{\phi}_{l}
\end{array}\right]=\frac{1}{N}\left[\begin{array}{c}
\dot{\theta}_{r m} \\
\dot{\theta}_{l m}
\end{array}\right] .
$$

The motors' torques, $\tau_{s r}$ and $\tau_{s l}$, are related to the wheels' torques, $\tau_{r}$ and $\tau_{l}$, as

$$
\left[\begin{array}{c}
\tau_{r} \\
\tau_{l}
\end{array}\right]=N\left[\begin{array}{c}
\tau_{s r} \\
\tau_{s l}
\end{array}\right]
$$

The relationship between the angular wheel velocities and the velocity vector $\mathbf{v}$ is given by

$$
\left[\begin{array}{c}
\dot{\phi}_{r} \\
\dot{\phi}_{l}
\end{array}\right]=\frac{1}{r}\left[\begin{array}{cc}
1 & R \\
1 & -R
\end{array}\right]\left[\begin{array}{c}
v \\
\omega
\end{array}\right] .
$$

Using equations (1)-(6), the dynamic equation of the wheelchair system, including actuator dynamics, can be written as

$$
\begin{aligned}
& {\left[\begin{array}{cc}
m & 0 \\
0 & I-m d^{2}
\end{array}\right]\left(\begin{array}{c}
\dot{v} \\
\dot{\omega}
\end{array}\right)+\left(\begin{array}{l}
\bar{\tau}_{d r} \\
\bar{\tau}_{d l}
\end{array}\right)=} \\
& \frac{N \cdot K_{T}}{R_{a}} \frac{1}{r}\left[\begin{array}{cc}
1 & 1 \\
R & -R
\end{array}\right]\left(\begin{array}{l}
u_{r} \\
u_{l}
\end{array}\right)-\frac{N^{2} K_{T} K_{b}}{R_{a}} \frac{1}{r}\left[\begin{array}{cc}
1 & 1 \\
R & -R
\end{array}\right]\left(\begin{array}{c}
v \\
\omega
\end{array}\right)
\end{aligned}
$$

where $K_{T r}=K_{T l}=K_{T} ; K_{b r}=K_{b l}=K_{b} ; R_{a r}=R_{a l}=$ $R_{a}$.

Without loss of generality, the dynamic equation of the wheelchair system, equation (7), can also be expressed by the following

$$
\left\{\begin{array}{l}
v=g_{11}(s) u_{v}+g_{12}(s) u_{\omega} \\
\omega=g_{21}(s) u_{v}+g_{22}(s) u_{\omega}
\end{array}\right.
$$

where $u_{v}$ and $u_{\omega}$ are reference input voltages and $g_{i j}(s)$ with $i, j=1,2$ is the element of system transfer matrix $G(s)$.

$$
G(s)=\left[\begin{array}{ll}
g_{11}(s) & g_{12}(s) \\
g_{21}(s) & g_{22}(s)
\end{array}\right]+\left[\begin{array}{ll}
\Delta g_{11}(s) & \Delta g_{12}(s) \\
\Delta g_{21}(s) & \Delta g_{22}(s)
\end{array}\right]
$$

with $g_{i j}(s)=\frac{K_{i j}}{\left(1+s T_{i j}\right)} e^{-\tau_{i j} s} ; \Delta g_{i j}(s)$ is system uncertainty.

The off-diagonal elements of the wheelchair dynamic model are coupling effects between input and output, implying that this model is non-symmetry. These coupling effects are subject to elimination using the proposed diagonalization technique. 


\section{B. Parameter verification procedure}

To verify wheelchair dynamics parameters, a pair input voltage is introduced to drive two corresponding direct-current motors. For each input pair $\left(u_{v}\right.$ and $\left.u_{\omega}\right)$, the corresponding outputs (velocities) are measured continuously for $10 \mathrm{~s}$. The voltage inputs are varied so that the outputs are tested in all their ranges. For each pair of input, the experiment is conducted five times to measure variations of the dynamics parameters. Due to the model variations, the responses of the wheelchair dynamic model will vary from a lower-bounded to an upper-bounded model. After intensively testing output responses with different control input pairs, parameters of wheelchair dynamics are obtained as in the following ranges:

$$
\begin{aligned}
& K_{11} \in[1.0 ; 1.80] ; T_{11} \in[0.55 ; 1.05] ; \tau_{11} \in[0.1 ; 0.35] \\
& K_{21} \in[0.1 ; 0.26] ; T_{21} \in[0.30 ; 0.40] ; \tau_{21} \in[0.1 ; 0.30] \\
& K_{12} \in[0.1 ; 0.25] ; T_{12} \in[0.35 ; 0.45] ; \tau_{12} \in[0.1 ; 0.20] \\
& K_{22} \in[1.0 ; 2.60] ; T_{22} \in[0.45 ; 0.55] ; \tau_{22} \in[0.1 ; 0.30] .
\end{aligned}
$$

As a result, equations for three dynamic model equations of the wheelchair system are obtained as follows

$$
\begin{aligned}
& G_{0}(s)=\left[\begin{array}{ll}
\frac{1.400}{(1+0.800 s)} e^{-0.225 s} & \frac{0.125}{(1+0.400 s)} e^{-0.150 s} \\
\frac{0.100}{(1+0.350 s)} e^{-0.200 s} & \frac{1.800}{(1+0.500 s)} e^{-0.200 s}
\end{array}\right] \\
& G_{1}(s)=\left[\begin{array}{ll}
\frac{1.800}{(1+0.550 s)} e^{-0.100 s} & \frac{0.220}{(1+0.350 s)} e^{-0.100 s} \\
\frac{0.250}{(1+0.300 s)} e^{-0.100 s} & \frac{2.600}{(1+0.450 s)} e^{-0.100 s}
\end{array}\right] \\
& G_{2}(s)=\left[\begin{array}{ll}
\frac{1.000}{(1+1.050 s)} e^{-0.350 s} & \frac{0.040}{(1+0.450 s)} e^{-0.200 s} \\
\frac{0.005}{(1+0.400 s)} e^{-0.300 s} & \frac{1.000}{(1+0.550 s)} e^{-0.300 s}
\end{array}\right]
\end{aligned}
$$

where $G_{0}(s)$ is the nominal dynamic model, and $G_{1}(s)$ and $G_{2}(s)$ are the upper model and the lower model respectively. Note that the model does not assume symmetry in the motors and physical plant of the wheelchair.

\section{Diagonalization techniQue}

When designing a controller for a multivariable feedback system, it is crucial to consider the following four criteria: ease of the design procedure; computational burden in the design procedure; the complexity of the compensator; and the quality of a closed-loop performance. In consideration of these criteria, a diagonalization technique aims at reducing the multivariable design problem to a series of independent scalar closed-loop design problems. The diagonalization technique consists of two phases: triangularization phase and diagonalization phase.

\section{A. Triangularization with TDD property}

For a given proper $(n x n)$ square plant $G(s)$, the purpose of this stage is to search for the compensation scheme so that the compensated plant is triangular-diagonal-dominant $(T D D)$. A uni-modular pre-compensator matrix $U(s)$ can always be constructed over the principal ideal domain $\Gamma$ of a proper plant transfer function matrix $G(s)$ such that

$$
T(s)=G(s) \cdot U(s)
$$

where $T(s)$ is triangular.

The following steps are used to construct $T(s)$

Step 1: Move the lowest degree element in the first row to the (1-1) position,

Step 2: Subtract a multiple of the first column from the second, third, $\cdots, n$-th column to guarantee $\delta\left(g_{1 i}\right)<\delta\left(g_{11}\right)$, with $i>1$,

Step 3: If one or more of $g_{1 i}(s)$ is nonzero, then return to step 1, otherwise move to step 4,

Step 4: Temporarily delete the first row and column,

Step 5: Repeat the procedure of step 1 to step 4 on the remaining matrix until a lower triangular structure is obtained. This leaves the temporarily deleted rows and column unaltered.

After obtaining $T(s)$, we need to check whether $T(s)$ is a $T D D$ matrix. Sufficient conditions for guaranteeing the $T D D$ property are outlined in the Lemma 6 in [19].

\section{B. Diagonalization}

In this phase, the row-normalized matrix $A(s)$ of the triangular matrix $T(s)$ is designated as follows

$$
A(s)=\tilde{T}^{-1}(s) T(s)
$$

where $\tilde{T}=\operatorname{diag}\left[t_{11}(s), t_{22}(s), \cdots, t_{n n}(s)\right]$.

Theorem 1: Let $G(s)$ be a proper transfer function matrix and $T(s)$ be the compensated plant with triangular structure derived from $G(s)$ above. If the row-normalized matrix $A(s)$ for the triangular matrix is stable (not necessary proper) then there exists a pre-compensator $V(s)$ such that

$$
D(s)=G(s) U(s) V(s)
$$

with $D(s)$ is proper and diagonal, and $V(s)$ is found as $V(s)= \begin{cases}A^{-1}(s) & \text { if } A(s) \text { is stable and proper } \\ A^{-1}(s) X^{-1}(s) & \text { if } A(s) \text { is stable and not proper }\end{cases}$ where $X(s)$ is a diagonal polynomial matrix chosen so that $A^{-1}(s) X^{-1}(s)$ is stable and proper.

\section{Proof}

If $A(s)$ is stable and proper then

$$
D(s)=G(s) U(s) V(s)=T(s)\left[\tilde{T}^{-1}(s) \cdot T(s)\right]^{-1}=\tilde{T}(s) .
$$

If $A(s)$ is stable and not proper then

$$
\begin{aligned}
D(s)=G(s) U(s) V(s) & =T(s)\left[\tilde{T}^{-1}(s) \cdot T(s)\right]^{-1} X^{-1}(s) \\
& =\tilde{T}(s) \cdot X^{-1}(s) .
\end{aligned}
$$

Therefore, it is clear that $D(s)$ has the required properties.

\section{Neural Network Control Design}

Consider an uncertain discrete-time system with a single input and single output as follows

$$
\left\{\begin{array}{l}
x(k+1)=\left(A_{d}+\Delta A_{d}\right) x(k)+\left(B_{d}+\Delta B_{d}\right) u(k) \\
y(k)=C_{d} x(k) ; x(0)=x_{0} .
\end{array}\right.
$$

where uncertainty matrices, $\Delta A_{d}$ and $\Delta B_{d}$, are assumed to be bounded. 
The optimal neural network control scheme for system (12) is proposed as follows in Fig.2. For a given $(n, m, 1)$ structure in Fig. 3 with $n$ input nodes, $m$ hidden nodes and 1 output node, the output of the neural network can be calculated as follows

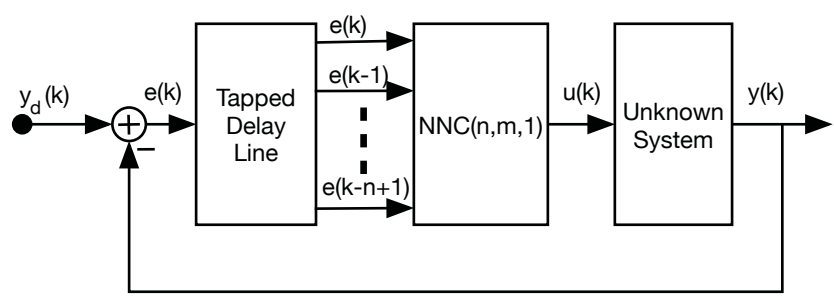

Fig. 2. Neural network control scheme for an uncertain scalar system.

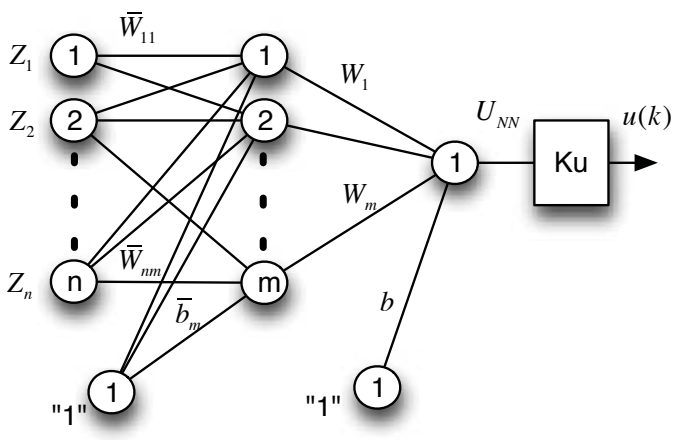

Fig. 3. Neural Network control structure.

$$
U_{N N}=f_{2}\left(\sum_{j=1}^{m} W_{j} f_{1}\left(\sum_{i=1}^{n} \bar{W}_{i j} Z_{i}+\bar{b}_{j}\right)+b\right)
$$

where $W_{j}$ is the weight between a $j^{t h}$ hidden node and an output node, $\bar{W}_{i j}$ is the weight between a $i^{\text {th }}$ input node and a $j^{\text {th }}$ hidden node, $\bar{b}_{j}$ is the $j^{\text {th }}$ bias of the hidden layer, $b$ is the bias of the output layer, and $f_{1}$ and $f_{2}$ are the activation function of the output-hidden layer and hidden-input layer, respectively.

$$
f_{1}(n)=\frac{1-e^{-2 n}}{1+e^{-2 n}} ; f_{2}(n)=n .
$$

Updating rules for optimal neural network controller: The objective here is to design a control law so that the output $y(k+1)$ tracks the reference $y_{d}(k+1)$ with the greatest accuracy. In other words, the control objective is to construct a control law which enables the system error, $e(k+1)=y_{d}(k+1)-y(k+1)$, to converge to zero, or at least to an acceptable tolerance with a desired finite time of $T_{0}$. The cost function of the neural network controller is designated by the following equation:

$$
E=\frac{1}{2}\left(y_{d}(k+1)-y(k+1)\right)^{2}=\frac{1}{2} e^{2}(k+1) .
$$

To minimize the cost function $\mathrm{E}$, it is necessary to change the weights of the neural network controller to the direction of a negative gradient. Applying the chain rule, the resulting functions are obtained as

$\Delta \theta=-\eta \frac{\partial E}{\partial \theta}=-\eta \frac{\partial E}{\partial y} \frac{\partial y}{\partial u} \frac{\partial u}{\partial U_{N N}} \frac{\partial U_{N N}}{\partial \theta}=\eta e(k) K_{u} \frac{\partial y}{\partial u} \frac{\partial U_{N N}}{\partial \theta}$

where $\theta$ is neural network weights and $\eta$ is learning rate.

The Jacobian of the plant is defined and calculated directly from the plant as follows:

$$
J(k)=\frac{\partial y(k)}{\partial u(k)}=-C_{d}\left(A_{d}+\Delta A_{d}\right)^{-1}\left(B_{d}+\Delta B_{d}\right) .
$$

Define

$$
\begin{gathered}
S_{0}=f_{1}\left(\sum_{i=1}^{n} \bar{W}_{i j} Z_{i}+\bar{b}_{j}\right) ; S_{1}=f_{1}{ }^{\prime}\left(\sum_{i=1}^{n} \bar{W}_{i j} Z_{i}+\bar{b}_{j}\right) \\
S_{2}=f^{\prime}{ }_{2}\left(\sum_{j=1}^{m} W_{j} f_{1}\left(\sum_{i=1}^{n} \bar{W}_{i j} Z_{i}+\bar{b}_{j}\right)+b\right) .
\end{gathered}
$$

For a given neural network structure, updating rules for its weights are shown as follows:

$$
\begin{aligned}
& W_{j}(k+1)=W_{j}(k)+\alpha \delta(k) S_{2} S_{0} \\
& b_{j}(k+1)=b_{j}(k)+\alpha \delta(k) S_{2} \\
& \bar{W}_{i, j}(k+1)=\bar{W}_{i, j}(k)+\eta \delta(k) S_{2} S_{1} W_{j} Z_{i} \\
& \bar{b}_{j}(k+1)=\bar{b}_{j}(k)+\eta \delta(k) S_{2} S_{1} W_{j},
\end{aligned}
$$

where the small positive $\alpha$ and $\eta$ represent the learning rates for the output-hidden layer and the hidden-input layer, respectively, and $\delta(k)=e(k) K_{u} \frac{\partial y(k)}{\partial u(k)}$.

\section{REAL-TIME EXPERIMENTAL RESULTS AND DISCUSSION}

\section{A. Diagonal Decoupling Control Design}

\section{Triangularization of wheelchair system}

Step 1: In the first row, the element $(1,1)$ already has the lowest degree $(\delta=2)$.

Step 2: Subtract a multiple of the first column from the second to ensure $\delta\left(g_{12}\right)<\delta\left(g_{11}\right)$

$$
\frac{0.125}{(1+0.4 s)(1+0.15 s)}=a\left(\frac{1.4}{(1+0.8 s)(1+0.225 s)}\right)+b
$$

where

$$
a=\left(\frac{0.125}{1.4}\right)\left(\frac{(1+0.8 s)(1+0.225 s)}{(1+0.4 s)(1+0.15 s)}\right) ; b=0 .
$$

The compensator is therefore chosen as

$$
U(s)=\left[\begin{array}{cc}
1 & -\left(\frac{0.125}{1.4}\right)\left(\frac{(1+0.8 s)(1+0.225 s)}{(1+0.4 s)(1+0.15 s)}\right) \\
0 & 1
\end{array}\right] .
$$

The resulting decoupled transfer function matrix $T(s)$ can be calculated as follows

$$
\begin{aligned}
& T(s)=G_{0}(s) \cdot U(s)= \\
& {\left[\begin{array}{cc}
\frac{1.4}{0.18 s^{2}+1.025 s+1} & 0 \\
\frac{0.07 s^{2}+0.55 s+1}{0.07 s^{2}} & \frac{17.06 s+117.1}{s^{3}+13.67 s^{2}+56.67 s+66.67}
\end{array}\right] .}
\end{aligned}
$$




\section{Diagonalization of wheelchair system}

Using theorem 1, the row-normalized matrix $A(s)$

$$
A=\tilde{T}^{-1} T=\left[\begin{array}{cc}
1 & 0 \\
9 \frac{100 s^{3}+1367 s^{2}+5667 s+6667}{(853 s+5855)\left(7 s^{2}+55 s+100\right)} & 1
\end{array}\right] .
$$

Obviously, $A(s)$ is stable and proper. Thus, the precompensator $V(s)=A^{-1}(s)$ is obtained as

$$
V(s)=\left[\begin{array}{cc}
1 & 0 \\
-9 \frac{100 s^{3}+1367 s^{2}+5667 s+6667}{(853 s+5855)\left(7 s^{2}+55 s+100\right)} & 1
\end{array}\right] .
$$

The nominal compensated model for the wheelchair is depicted as

$$
\begin{aligned}
& D_{0}(s)=G_{0}(s) U(s) V(s)=T(s) V(s)=\tilde{T}(s) \\
& =\left[\begin{array}{cc}
\frac{1.4}{0.18 s^{2}+1.025 s+1} & 0 \\
0 & \frac{17.06 s+117.1}{s^{3}+13.67 s^{2}+56.67 s+66.67}
\end{array}\right] .
\end{aligned}
$$

Finally, the wheelchair dynamics are decoupled into two uncertain scalar systems presented in (12) with following parameters:

The discrete linear velocity system with $T s=25 \mathrm{~ms}$ :

$$
\begin{aligned}
& A_{d}=\left[\begin{array}{cc}
0 & -0.8673 \\
1 & 1.8641
\end{array}\right] ; B_{d}=\left[\begin{array}{l}
0.00221109 \\
0.00231854
\end{array}\right] ; C_{d}=\left[\begin{array}{ll}
0 & 1
\end{array}\right] ; \\
& \Delta A_{d}=\left[\begin{array}{cc}
0 & \pm 0.123 \\
0 & \pm 0.129
\end{array}\right] ; \Delta B_{d}=\left[\begin{array}{l} 
\pm 0.064 \\
\pm 0.072
\end{array}\right] .
\end{aligned}
$$

The discrete angular velocity system with $T s=25 \mathrm{~ms}$

$$
\begin{aligned}
& A_{d}=\left[\begin{array}{ccc}
0 & 0 & 0.710526 \\
1 & 0 & -2.39155 \\
0 & 1 & 2.68015
\end{array}\right] ; B_{d}=\left[\begin{array}{c}
-0.00401019 \\
0.0005167 \\
0.00503874
\end{array}\right] \\
& A_{d}=\left[\begin{array}{lll}
0 & 0 & \pm 0.011 \\
1 & 0 & \pm 0.250 \\
0 & 1 & \pm 0.135
\end{array}\right] ; \Delta B_{d}=\left[\begin{array}{c} 
\pm 0.00075 \\
\pm 0.00095 \\
\pm 0.0100
\end{array}\right] ; C_{d}=\left[\begin{array}{l}
0 \\
0 \\
1
\end{array}\right]^{T} .
\end{aligned}
$$

\section{B. Dynamic control}

Since the wheelchair dynamics is decoupled into two independent uncertain scalar systems, two neural network controllers are required to provide desired closed-loop control criteria: settling time $T s \leq 3 s$, overshoot $M_{p} \leq 5 \%$, and zero steady error to step inputs.

The training procedure presented in the previous section is used to train $N N C_{v}$ and $N N C_{\omega}$ controllers. The output of the neural controllers are limited in the interval $[-2.5,2.5]$, and the constant $K u$ is chosen to be 2.5 . The data used for training neural network controllers are actual data acquired from various experiments. The training epoch consists of 400 iterations, equivalent to $10 \mathrm{~s}$ with sampling time $T s=25$ $m s$. The data are divided into three sets: the training set, the validation set and the test set. The validation set is used to ensure that overtraining would not occur. The optimal structure of the neural network controllers and their corresponding weights are determined off-line. The optimal learning rates for the $N N C_{v}$ controller are chosen as $\alpha=0.015 ; \eta=0.01$, and its optimal structure is $(3,4,1)$, which is equivalent to 3 input nodes, 4 hidden nodes and 1 output node. Similarly, the optimal learning rate values for the $N N C_{\omega}$ controller are chosen as $\alpha=0.015 ; \eta=0.02$, and its optimal structure is $(3,5,1)$.

For validation, real-time experiments are implemented on the wheelchair system as shown in Fig. 4 using NI LabWindows CVI 2010. The linear velocity and angular velocity are filtered and measured using the USB4 Encoder Data Acquisition system from USDigital. We use the Ascension Flock of Birds magnetic position tracking system to measure the wheelchair position. The outputs of the neural controllers are sent autonomously to control the wheelchairs speed using the NI USB6009 interface. The asynchronous timers and multithreading technique are used to ensure real-time sampling of $25 \mathrm{~ms}$ can be achieved for the control loops. Although some human manipulated input such as joystick or head movement control exists, all of the experiments described below are autonomous driving experience against various set points and predefined desired paths.

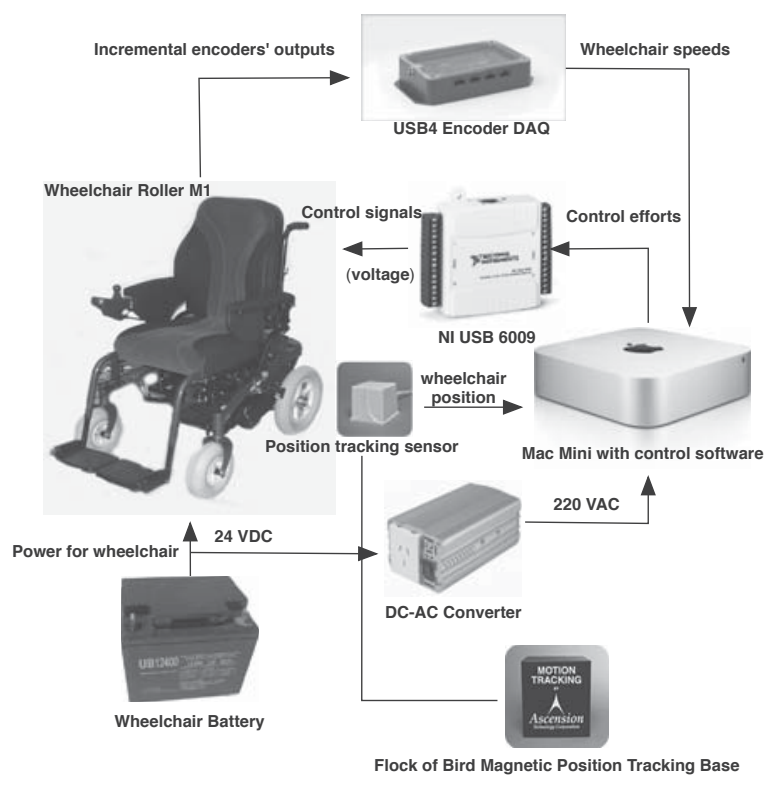

Fig. 4. Wheelchair system for real-time experiments.
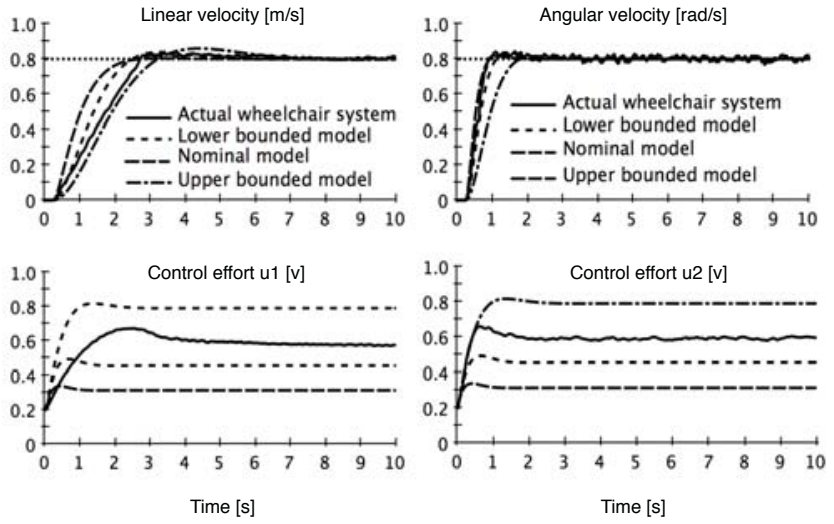

Fig. 5. Closed-loop responses of linear velocity and angular velocity loops. 

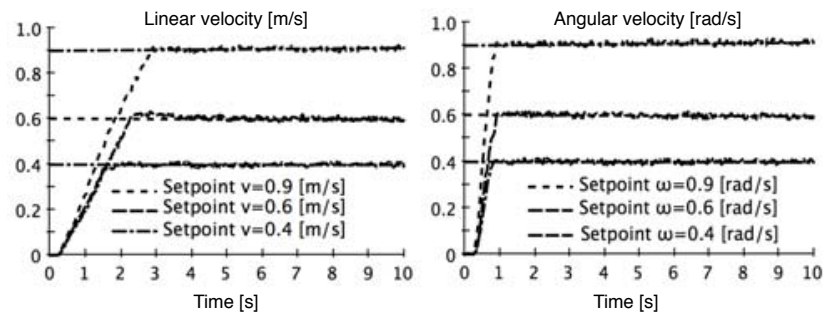

Fig. 6. Real-time closed-loop responses of linear velocity and angular velocity loops with different set-points.
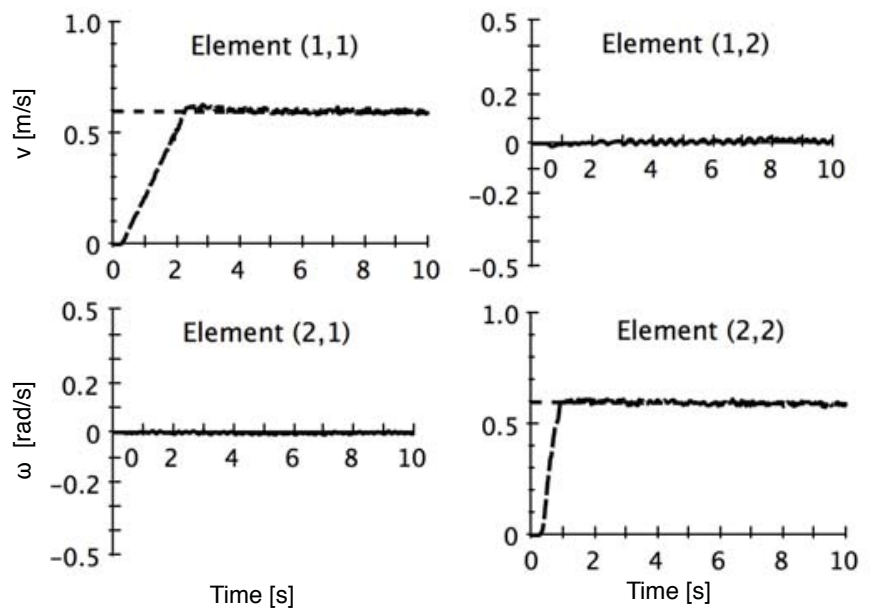

Time $[\mathrm{s}]$

Fig. 7. Real-time closed-loop responses of wheelchair dynamics, element $(1,2)$ and element $(2,1)$ show the decoupling effects between two loops are substantially reduced.

Experiment 1: This real-time implementation demonstrates the performance of the controlled system. The linear velocity and angular velocity are set to be $0.8 \mathrm{~m} / \mathrm{s}$ and $0.8 \mathrm{rad} / \mathrm{s}$ respectively, and the wheelchair carries a $75 \mathrm{~kg}$ person and travels on a wooden surface. Real-time results of the two velocity loops in Fig. 5 show that the proposed controllers achieve the desired performance $\left(T_{s} \leq 3 s, M_{p} \leq 5 \%\right.$, and zero steady-state error). The results also show that real-time responses of the two control loops are within the boundaries set by the upper bound and lower bound models.

Experiment 2: This real-time experiment shows the wheelchair performance when it travels on various surfaces while carrying a $60 \mathrm{~kg}$ person. Specifically, it travels on a wooden surface with a set-point pair $(0.4 \mathrm{~m} / \mathrm{s}, 0.4 \mathrm{rad} / \mathrm{s})$, on a cement surface $(0.6 \mathrm{~m} / \mathrm{s}, 0.6 \mathrm{rad} / \mathrm{s})$ and on a carpet surface $(0.9 \mathrm{~m} / \mathrm{s}, 0.9 \mathrm{rad} / \mathrm{s})$. Fig. 6 confirms that using the proposed controllers, optimal performances are achieved on various surfaces. The real-time implementation on a cement surface shown in Fig. 7 also demonstrates that the coupling effects between the two control loops are substantially reduced.

Experiment 3: A multivariable PID (MPID) method based on a combination of PID controllers and diagonalization technique has been introduced for comparison. Two PI controllers are independently designed for the linear velocity loop and angular velocity loop. Using the root-locus technique, the
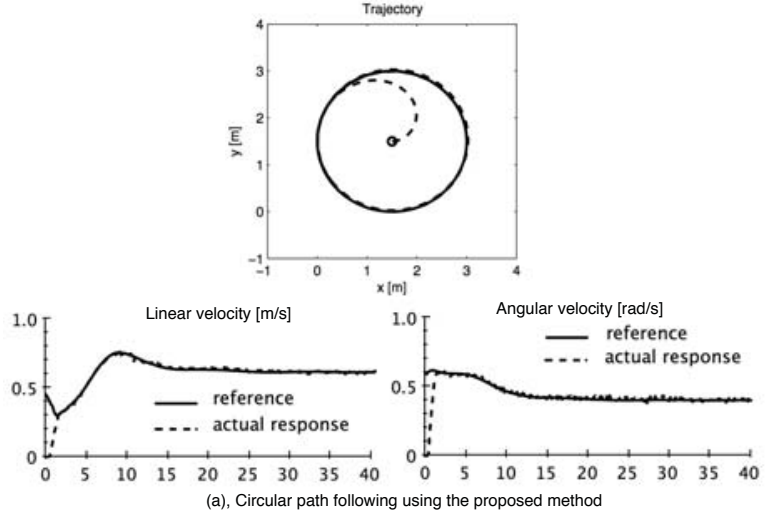

Träectory
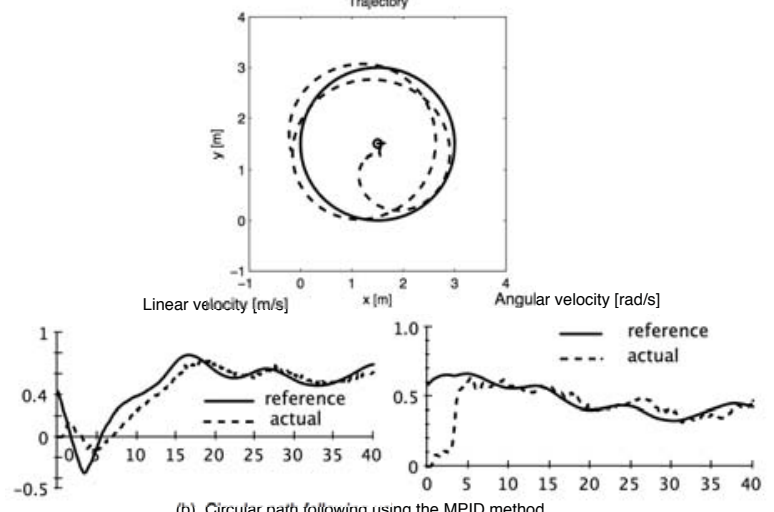

Fig. 8. Real-time circular path following tasks.

Linear velocity $[\mathrm{m} / \mathrm{s}]$

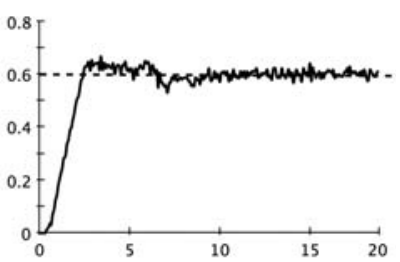

(a) Adaptive line tracking on uneven and rough surface using the proposed method
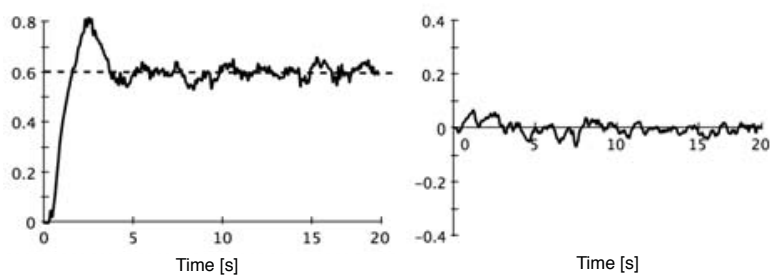

(b) Adaptive line tracking on uneven and rough surface using the MPID method

Fig. 9. Real-time adaptive line tracking tasks on uneven and rough surface.

PI parameters for the linear velocity loop are chosen as ( $\left.K_{p}=0.22 ; K_{i}=0.55\right)$ while those for the angular velocity loop are $\left(K_{p}=0.15 ; K_{i}=0.25\right)$. In this task, the wheelchair carrying an $85 \mathrm{~kg}$ person travels on a rough cement surface (outdoor environment). Compared to the MPID method, the real-time results in Fig. 8 show that the performance obtained by the proposed method is better in terms of response time, tracking 
accuracy and robustness.

Experiment 4: This real-time experiment aims to test the robustness of the controlled system against external disturbances. In this experiment, the wheelchair carrying a $75 \mathrm{~kg}$ person and travels on an uneven and rough surface. Compared to the MPID method, the results in Fig. 9 confirm that the proposed approach achieves better and more robust performance.

\section{CONCLUSION}

In this paper, an advanced neural-network-based diagonal decoupling control method has been developed and implemented for a powered wheelchair system. This control method is based on a combination of diagonalization technique and neural network control design. The advantage of this novel approach is that it removes the interaction in the nominal multivariable model, and provides a robust solution for both parameter uncertainties and external disturbances. Using the diagonalization technique, a stable multivariable decoupler is found and this multivariable system is reduced to a series of scalar control problems. These scalar control problems are then solved using an optimal neural network control design technique.

For this wheelchair, an uncertain multivariable model is obtained using a parameter verification procedure. This consists of a nominal dynamic model, completed with upper bound and lower bound models. A multivariable decoupler and two independent optimal neural network controllers are developed. Essentially, using the obtained dynamic model, the problem of the plant's Jacobian calculation is eliminated in this neural network controller design.

The real-time experimental results show that the interactions between the two subsystems are eliminated for the nominal system with the implementation of the multivariable decoupler. This is particularly important as the control performance of the wheelchair will be consistent even when this system diverts from its nominal condition, including when it is operating on an uneven environment. It can be seen that the control robustness of the system has been achieved, as although the actual performance of the wheelchair diverts from the performance effected by the expected nominal model, it is still contained within those generated by the lower bound and upper bound models. The results also show that excellent overall system performance is achieved for an adaptive linepath tracking task when the wheelchair operates on different surfaces with external disturbances. In addition, excellent circular path-following control capability of the system is validated employing users with various weights. Therefore, real-time experimental results confirm that the robust performance of this multivariable wheelchair control system can be achieved under model uncertainties and unknown external disturbances.

\section{REFERENCES}

[1] R. Cooper, M. Boninger, D. Spaeth, D. Ding, S. Guo, A. Koontz, S. Fitzgerald, R. Cooper, A. Kelleher, and D. Collins, "Engineering better wheelchairs to enhance community participation," IEEE Transactions on Neural Systems and Rehabilitation Engineering, vol. 14, pp. $438-$ 455, Dec 2006.
[2] R. Fierro and F. Lewis, "Control of a nonholonomic mobile robot using neural networks," IEEE Transactions on Neural Networks, vol. 9, pp. 589 -600 , Jul 1998.

[3] R. Cooper, L. Widman, D. Jones, R. Robertson, and I. Ster, J.F., "Force sensing control for electric powered wheelchairs," IEEE Transactions on Control Systems Technology, vol. 8, pp. 112 -117, Jan 2000.

[4] R.-X. Chen, L.-G. Chen, and L. Chen, "System design consideration for digital wheelchair controller," IEEE Transactions on Industrial Electronics, vol. 47, pp. 898 -907, Aug 2000.

[5] A. Argyros, P. Georgiadis, P. Trahanias, and D. P. Tsakiris, "Semiautonomous navigation of a robotic wheelchair," Journal of Intelligent and Robotic Systems, vol. 34, pp. 315-329, Jul 2002.

[6] F. Fujii and K. Wada, "For a better maneuverability of the electrically powered wheelchair - a pilot-model based approach," in Proceedings of 2003 International Symposium on Micromechatronics and Human Science, pp. 125 - 132, Oct 2003.

[7] L. Boquete, R. Barea, R. García, M. Mazo, and M. A. Sotelo, "Control of a robotic wheelchair using recurrent networks," Journal of Autonomous Robots, vol. 18, pp. 5-20, Jan 2005.

[8] Q. Zeng, B. Rebsamen, E. Burdet, and C. L. Teo, "A collaborative wheelchair system," IEEE Transactions on Neural Systems and Rehabilitation Engineering, vol. 16, pp. 161 -170, Apr 2008.

[9] H. T. Nguyen and S. W. Su, "Conditions for triangular decoupling control," International Journal of Control, vol. 82, pp. 1575-1581, Sep 2009.

[10] V. Chen and Y.-H. Pao, "Learning control with neural networks," in IEEE International Conference on Robotics and Automation, vol. 3, pp. 1448 -1453 , May 1989.

[11] K. Narendra and K. Parthasarathy, "Identification and control of dynamical systems using neural networks," IEEE Transactions on Neural Networks, vol. 1, pp. 4 -27, Mar 1990.

[12] D. Psaltis, A. Sideris, and A. Yamamura, "A multilayered neural network controller," IEEE Control Systems Magazine, vol. 8, pp. 17 -21, Apr 1988.

[13] L. Acosta, J. A. Méndez, S. Torres, L. Moreno, and G. N. Marichal, "On the design and implementation of a neuromorphic self-tuning controller," Neural Process Letters, vol. 9, pp. 229-242, Jun 1999.

[14] M. Saerens and A. Soquet, "Neural controller based on back-propagation algorithm," IEE Proceedings F Radar and Signal Processing, vol. 138, pp. $55-62$, Feb 1991.

[15] G. W. Ng and P. A. Cook, "On-line adaptive control of non-linear plants using neural networks with application to liquid level control," International Journal of Adaptive Control and Signal Processing, vol. 12, pp. 13-28, Dec 1998.

[16] K. P. Venugopal, R. Sudhakar, and A. S. Pandya, "An improved scheme for direct adaptive control of dynamical systems using backpropagation neural networks," Journal of Circuits, Systems Signal Processing, vol. 14, pp. 213-236, Feb 1995.

[17] T. N. Nguyen, S. Su, and H. Nguyen, "Robust neuro-sliding mode multivariable control strategy for powered wheelchairs," IEEE Transactions on Neural Systems and Rehabilitation Engineering, vol. 19, pp. 105 -111 , Feb 2011.

[18] T. Das and I. Kar, "Design and implementation of an adaptive fuzzy logic-based controller for wheeled mobile robots," IEEE Transactions on Control Systems Technology, vol. 14, pp. 501 - 510, May 2006.

[19] N. Hung and B. Anderson, "Triangularization technique for the design of multivariable control systems," IEEE Transactions on Automatic Control, vol. 24, pp. 455 - 460, Jun 1979.

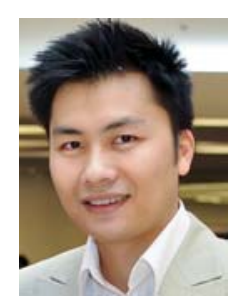

Tuan Nghia Nguyen received his $\mathrm{PhD}$ in Engineering from the University of Technology, Sydney (UTS) in March 2010. He was awarded as a recipient of the Chancellor's List in recognition of exceptional scholarly achievement in $\mathrm{PhD}$ research in 2010 . Currently, he is a Chancellors Postdoctoral Research Fellow in the Centre for Health Technologies, Faculty of Engineering at the UTS. His current research interests include biomedical engineering, advanced control and artificial intelligence for health technologies. He is also a member of the Institution of Electrical and Electronics Engineers (IEEE) and the Institution of Engineers, Australia (IEAust). 
Steven Su obtained his PhD degree from the Aus-
tralian National University (ANU) in 2002. From

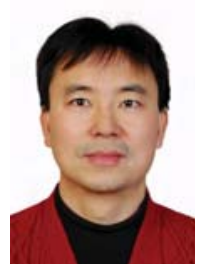
2002 to 2004, he was a postdoctoral research fellow in the School of CEIC, the University of New South Wales (UNSW). He was also a research fellow in the School of EET, UNSW from 2004 to 2006.

$\mathrm{He}$ is currently a senior lecturer in the Faculty of Engineering and IT, the University of Technology, Sydney (UTS). His main research interests include biomedical system modelling and control, multiple model adaptive control, fault tolerant control, computational intelligence, and wearable monitoring systems.

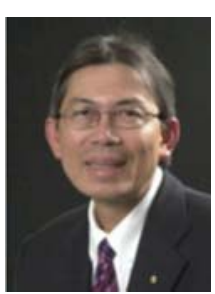

Hung T Nguyen is a Professor of Electrical Engineering at the University of Technology, Sydney (UTS). He is Dean of the Faculty of Engineering and Information Technology and Director of the Centre for Health Technologies at UTS. He received his $\mathrm{PhD}$ degree in 1980 from the University of Newcastle in Australia. His research interests include biomedical engineering, advanced control and artificial intelligence. He has developed biomedical devices for diabetes, disability, and cardiovascular diseases. He is a senior member of the Institute of Electrical and Electronic Engineers, and a Fellow of the Institution of Engineers, Australia and the British Computer Society. 